\title{
Ski and SnoN, potent negative regulators of TGF- $\beta$ signaling
}

\author{
Julien Deheuninck ${ }^{1}$, Kunxin Luo ${ }^{1}$ \\ ${ }^{1}$ UC Berkeley, Department of Molecular and Cellular Biology, 16 Barker Hall, MC3204, Berkeley, CA 94720, USA
}

\begin{abstract}
Ski and the closely related SnoN were discovered as oncogenes by their ability to transform chicken embryo fibroblasts upon overexpression. While elevated expressions of Ski and SnoN have also been reported in many human cancer cells and tissues, consistent with their pro-oncogenic activity, emerging evidence also suggests a potential anti-oncogenic activity for both. In addition, Ski and SnoN have been implicated in regulation of cell differentiation, especially in the muscle and neuronal lineages. Multiple cellular partners of Ski and SnoN have been identified in an effort to understand the molecular mechanisms underlying the complex roles of Ski and SnoN. In this review, we summarize recent findings on the biological functions of Ski and SnoN, their mechanisms of action and how their levels of expression are regulated.
\end{abstract}

Keywords: SnoN, Ski, Signal transduction, development, tumorigenesis, TGF- $\beta$ signaling, senescence

Cell Research (2009) 19:47-57. doi: 10.1038/cr.2008.324; published online 30 December 2008

\section{Introduction}

The sno gene (Ski novel gene) was initially discovered on the basis of its close homology to v-ski, the transforming component of the Sloan-Kettering virus (SKV) [1] and its cellular homolog c-ski [2]. The human Ski and SnoN proteins share an overall 50\% amino acid (aa) homology (and 36\% identity) with similar domain structures (Figure 1). The full-length sno gene encodes the 684-amino-acids SnoN protein as well as some reported alternatively spliced forms. These include SnoN2 (or mSno-dE3), resulting from a 46-aa deletion in exon 3, SnoI, which contains only the first 399 residues of SnoN, and SnoA, which differs from SnoN after exon 1 with a specific and shortened C-terminal end (Figure 1) [2-5]. The ski gene has not been shown to be regulated by alternative splicing.

Ski and SnoN are members of the Ski family that also contains two recently described members: Fussel-18 (Functional Smad suppressing element on chromosome 18) [6] and Fussel-15 (or LBXCOR1) [7]. Both Fussel18 and Fussel-15 have significant homology with Ski and SnoN, and can interact with R-Smads in transfected cells and inhibit TGF- $\beta$ or BMP responsive gene expression in reporter assays. Unlike Ski and SnoN, the expres-

Correspondence: Kunxin Luo

E-mail:kluo@berkeley.edu sion of Fussel-18 and Fussel-15 appears to be limited to neuronal tissues, suggesting that their functions may be more restricted.

Ski and SnoN contain several structural domains (Figure 1), including the DHD (Dachshund homology domain, also called DS domain for DACH, Ski and Sno) in their N-terminal region $[8,9]$ and a Smad4 binding domain [10]. The 100-aa DHD forms a compact, globular structure consisting of mixed alpha helix and beta sheets [11] that has features found in the forkhead/winged-helix family of DNA-binding proteins. Since neither Ski nor SnoN has been shown to directly bind DNA, the DHD may function to mediate interaction of Ski/SnoN with other proteins, such as the transcriptional co-regulator $\mathrm{N}-\mathrm{CoR}$ (nuclear receptor co-repressor) [12]. Close to the DHD domain stands an 80-aa $\mathrm{C} 2 \mathrm{H} 2 \mathrm{Zn}$-binding module with structural homology to the SAND domain (Sp100, AIRE1, NucP41/75 and DEAF1) [10]. This domain is only present in Ski, SnoN and Fussels, and is necessary for interacting with Smad4 [10]. The C-terminal regions of Ski and SnoN are less conserved, but may allow Ski and SnoN to homo- or hetero-dimerize through a coiledcoil motif located in this region $[13,14]$.

\section{Biological functions of Ski and SnoN}

In embryonic development

The ski and sno genes, although highly homologous, are not functionally redundant during development. In 

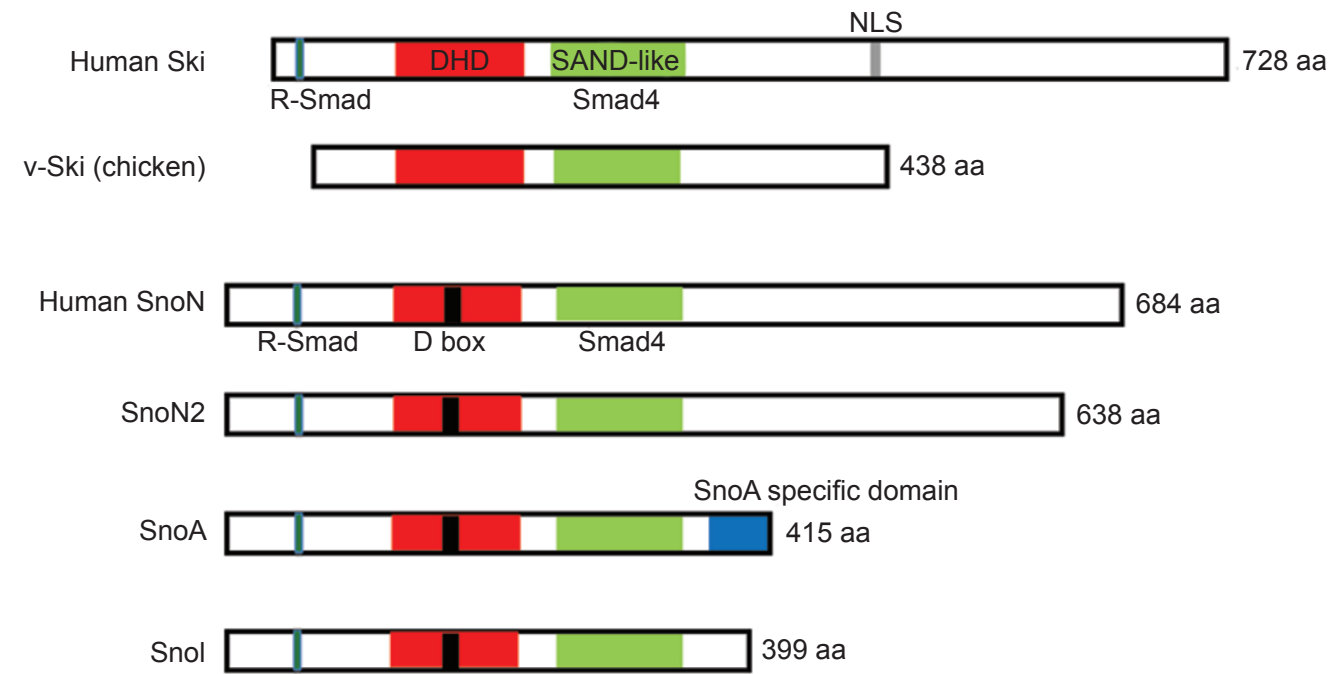

Figure 1 Domain structure of Ski and SnoN family of proteins. The number of amino acids for each protein is indicated on the right. DHD: Dachshund homology domain. SAND: Sp100, AIRE1, NucP41/75 and DEAF1. NLS: Nuclear localization sequence. $\mathrm{D}$ box: Degradation box. Explanations for domain functions are given in the main text.

vivo studies in Xenopus oocytes, zebrafish and mice have demonstrated a critical role of $s k i$ in the development of neuronal and muscle lineages [15-17]. In Xenopus, the XSki protein is maternal, and is maintained at a high level until the late neurula stage [18]. When injected into the Xenopus embryos, Ski was found to induce secondary neural axis formation and neural specific gene expression in ectoderm explants [17]. In mouse, Ski is expressed in all normal adult and in embryonic tissues at a low level [19]. Its expression is increased from E8.5 to E9.5 and from E12.5 to E15.5 as well as in some tumor cells in the adult. The first peak of Ski expression during embryogenesis (E8.5-9.5) correlates with neural tube closure and migration of neural crest cells, and the second peak overlaps with skeletal muscle development (E12.5-E15.5). This expression pattern indicates that Ski may play a role in the development of muscle, craniofacial structure, central and peripheral nervous systems, and respiratory tissues in the mouse embryo [20]. Supporting this notion, ski-null pups suffer from exencephaly, the absence of the cranial vault, due to failed neural tube closure and die perinatally [16]. In addition, these fetuses display facial clefting of varying severity and other craniofacial and skeletal abnormalities. The ski-null mice also show a marked decrease in skeletal muscle mass, probably due to a defect in secondary myoblast proliferation and/or survival [16]. The human ski gene is located on chromosome 1p36.3, and monosomy of this region of chromosome 1 results in the 1p36 syndrome, a genetic disorder characterized by craniofacial defects including a tower skull with a large and late closing anterior fontanel and a prominent forehead [21]. Interestingly, the phenotypes of the ski-knockout mice mirror many symptoms of the human $1 \mathrm{p} 36$ syndrome [22], suggesting that deletion of the ski gene may be partially responsible for the disease. Three lines of sno-knockout mice have been generated and published. One knockout mouse was shown to die before implantation [23], while two other mice with deletion of either the first exon of the sno gene (thus lacking both the DHD and the Smad4 binding domain) or the sno promoter are viable without apparent developmental defects [24]. Since the cause of this discrepancy has not been determined, the function of SnoN in embryonic development remains unclear.

\section{In adult tissues}

Ski and SnoN are expressed in virtually all adult tissues, albeit at low levels $[2,3,9]$. They can regulate the differentiation of several cell types, particularly those in neural and muscle lineages. In chicken and quail embryo fibroblasts, overexpression of either WT Ski or SnoN induces muscle differentiation $[25,26]$. In mice, loss of $s k i$ leads to reduced skeletal muscle mass [16, 22], whereas Ski overexpression causes muscle hypertrophy [27]. Consistent with these results, Ski and SnoN have been shown to activate the expression of muscle-specific genes $[28,29]$. In a tissue culture setting, SnoN also regulates neuronal cell function, for example as a potent promoter of axonal growth [30]. Similarly, Ski has been implicated in regulation of Schwann cell proliferation and myelina- 
tion [31]. In addition to muscle and neuronal lineages, Ski has also been shown to influence the growth and differentiation of hematopoietic cells in vitro [32, 33], and SnoN may be involved in regulation of T-cell function as two of the sno gene knockout mouse strains display defective T-cell activation [24]. Finally, the expression of Ski and SnoN is altered under many pathological conditions, including wound healing [34], liver regeneration [35] and obstructive nephropathy [36, 37]. The biological significance of these alterations is not clear. Although Ski and SnoN are expressed in all epithelial cells, their functions in normal epithelial cells have not been defined.

\section{In cancer progression}

The original discovery of Ski as the cellular homolog of a viral transforming protein leads to the classification of c-ski as a proto-oncogene. Indeed, early studies show that overexpression of Ski or SnoN promotes oncogenic transformation of avian fibroblasts as well as myogenic differentiation of quail embryo cells $[25,26,38]$. These early studies highlight an apparent contradiction, as Ski or SnoN overexpression favors either transformation or terminal differentiation, and hint at the potential complexity of Ski/SnoN action.
Supporting the pro-oncogenic properties of Ski and SnoN, expression of Ski and/or SnoN is elevated in many cancer cells and tissues, including those derived from esophageal squamous cell carcinoma [39-41], melanoma [42-44], estrogen receptor-positive breast carcinoma [45], colorectal carcinoma [46] and leukemia [33, 47]. Moreover, the human sno gene is located at $3 \mathrm{q} 26.2$, a chromosome locus frequently amplified in many tumors, including cancers of the lung [48, 49], esophagus [39], head and neck [50, 51], cervix $[49,52]$, ovary $[49,53]$ and prostate [54]. However, this chromosomal localization by itself is not sufficient to suggest a pro-oncogenic role of SnoN as the locus also contains many other genes that are known to promote cancer progression. The strongest evidence supporting the pro-oncogenic function of Ski or SnoN in mammalian tumorigenesis comes from the studies showing that reduction of SnoN expression by siRNA in human lung or breast cancer cells inhibits tumor growth both in vitro and in vivo [55], and that downregulation of Ski in pancreatic cancer cells also reduces tumor growth [56]. However, decreasing Ski expression by siRNA in lung and breast cancer cells has no effect on the transforming activity of these cells [55], suggesting that the tumor-promoting activity of Ski may
Pro-oncogenic

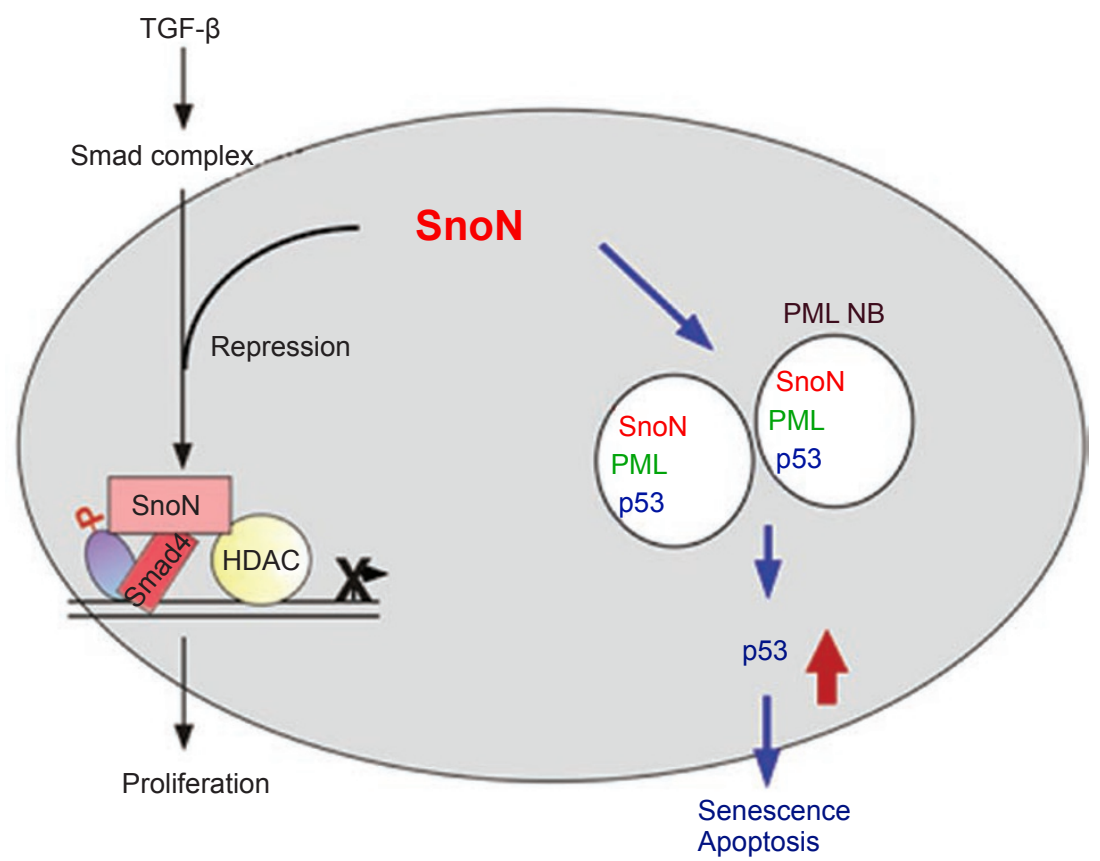

Figure 2 SnoN possesses both pro-oncogenic and anti-oncogenic activities. In the nucleus, SnoN can have either prooncogenic or anti-oncogenic activities through two pathways. SnoN may promote epithelial cell proliferation by antagonizing the growth-inhibitory activity of the TGF- $\beta / S$ mad pathway. Very high levels of SnoN may also trigger premature senescence through stabilizing p53 in a PML-dependent manner, and this pathway mediates the anti-oncogenic activity of SnoN. 
be restricted to certain types of cancers.

More recent surveys in human cancer tissues reveal a more complex pattern of Ski and SnoN expression during malignant progression. In tissue samples from patients with Barrett's esophagus, Ski and SnoN were found to be expressed at high levels in tissues with low grade dysplasia but absent in those with high grade dysplasia or adenocarcinoma [57]. In colorectal cancer with microsatellite instability, SnoN is downregulated in $39 \%$ of the samples and upregulated in $33 \%$ of the samples [58]. These results imply that high levels of Ski and SnoN may not always be beneficial to cancer cells. Indeed in recent years, more evidence has emerged indicating that both Ski and SnoN can act as tumor suppressors. First, heterozygous $\mathrm{sno}^{+/}[23]$ and $s k i^{+/}$[59] mice are more susceptible to carcinogen-induced tumors than WT mice, suggesting that an extra copy of the ski or sno gene protects against carcinogenesis in vivo. Recently, we showed that high levels of SnoN induce premature senescence through stabilization of p53. High levels of SnoN increase the transcription of PML, and through a physical interaction with the increased PML protein, SnoN is in turn recruited to the PML nuclear bodies, leading to stabilization of p53 (Figure 2). Consistent with a tumor suppressor function of senescence, overexpression of SnoN inhibits oncogene-induced transformation of primary MEF cells. More importantly, in a two-step skin carcinogenesis model, mice expressing a high level of SnoN were resistant to the development of papilloma and displayed senescence in vivo. This activity of SnoN is independent of its ability to antagonize the Smad proteins and provides a mechanistic basis for the tumor suppressor function of SnoN (Figure 2) [60]. Consistent with this tumor suppressor activity, the Drosophila dSno protein also inhibits cell growth [61]. Finally, downregulation of Ski or SnoN by shRNA enhances EMT of lung and breast cancer cells in vitro and tumor metastasis in vivo $[55,62]$. These data highlight the complex nature of Ski/SnoN functions in cancer, with both pro- and antioncogenic properties.

\section{Molecular mechanisms of Ski and SnoN action}

Ski and SnoN do not have catalytic activities, and therefore have to function through interaction with other cellular partners. Their primary function is related to transcriptional regulation, despite that they are unable to bind DNA directly. They can interact with a number of transcription factors, including Smads [63, 64], pRb [65], GATA1 [66], Gli3 [67] or retinoic acid receptor $\alpha(\mathrm{RAR} \alpha)$ $[32,47]$, and transcriptional co-regulators such as N-CoR [68] or $\mathrm{mSin} 3 \mathrm{~A}$ [12].
Negative regulation of TGF- $\beta$ signaling

So far the most important function described for Ski and $\mathrm{SnoN}$ is to negatively regulate TGF- $\beta$ signaling by binding to the Smad proteins [69]. Ski and SnoN interact simultaneously with the R-Smad ( $\operatorname{Smad} 2 / 3)$, through their N-terminal region, and with co-Smad (Smad4), through the SAND-like domain [10], and block the ability of the Smad complexes to activate transcription of TGF- $\beta$ target genes. A comparison of the crystal structure of the Smad4 binding domain of Ski in complex with Smad4 with that of the phospho-Smad2/Smad4 complex showed that Ski and phospho-Smad2 compete for binding to the same region on Smad4, predicting that binding of Ski to Smad4 results in the displacement of phosphoSmad2/3 from Smad4 and disruption of the functional Smad heteromeric complexes [10]. Indeed, biochemical analysis using Ski truncation mutants defective in binding to one of the Smad proteins confirmed this model [10]. Although both the R-Smads and Smad4 remain bound to Ski, they are no longer in an active heteromeric complex that could activate transcription [70]. Moreover, binding of Ski or SnoN may additionally stabilize the inactive Smad heteromer on DNA, possibly preventing further binding of active Smad complexes [70]. A later study showed that Smad3 could still be found in complex with Smad4 in the presence of a mutant Ski defective in binding to Smad3, arguing that the Smad complex may be inactivated instead of being disrupted [71, 72]. However, since not all the Smad complexes in the cells are bound to Ski, the detected Smad complex described above could belong to the Ski-independent population of Smads. Moreover, the above interactions were detected under overexpression conditions, and it is not clear whether this holds true under physiological conditions of Smad expression. In addition to disruption of the active Smad complexes, Ski or SnoN also prevents binding of the $\mathrm{R}$-Smads to transcriptional co-activator $\mathrm{p} 300 / \mathrm{CBP}[10$, 73] and actively recruits a transcriptional co-repressor complex containing N-CoR and HDAC to the targeted promoters $[63,64]$. It is likely that all these mechanisms function together to allow antagonism of Smad signaling by Ski/SnoN. High levels of Ski have also been reported to interfere with Smad2/3 phosphorylation by T $\beta R I$ [74]. Since Ski and SnoN are primarily located in the nucleus, any potential effect on receptor-mediated phosphorylation of $\mathrm{Smad} 2 / 3$ is unlikely to be direct.

Since Smads are critical mediators of the growth inhibitory signals of TGF- $\beta$ in epithelial cells, high levels of Ski or SnoN may promote cell proliferation through inhibiting the Smad proteins. Indeed, many human cancer cell lines express high levels of Ski or SnoN and are refractory to TGF- $\beta$-induced growth arrest $[42,55,75]$. 
Reducing Ski and/or SnoN expression in these cancer cells restores TGF- $\beta$ signaling and renders these cells sensitive to the growth inhibitory effects of TGF- $\beta$. These results suggest that the transforming activity of Ski or SnoN may be related to their ability to antagonize the Smads. Indeed, we have shown that at least in chicken embryo fibroblast cells where the oncogenic activity of Ski and SnoN was first defined, the ability of Ski and SnoN to bind to and repress the Smad proteins is required for their transforming activity [76]. In other pathological situations such as liver damage and regeneration where the growth inhibitory activity of TGF- $\beta$ needs to be suppressed, SnoN is found to be upregulated [35].

The TGF- $\beta$ superfamily of proteins, in particular BMP and activin play important roles in multiple aspects of vertebrate development. As Ski and SnoN also play important roles in embryonic development and in the differentiation of multiple cell lineages, an obvious question is whether these activities of Ski and SnoN are dependent on their ability to repress BMP or activin signaling. Interestingly, Ski, but not SnoN, can bind to and repress the activity of BMP-specific Smads, Smad1/5 [77, 78]. In Xenopus embryos, this ability of Ski to repress BMP signaling is required for the specification of neural cell fate [78]. Moreover, many craniofacial phenotypes found in the ski-null mice are reminiscent of those found in transgenic mice overexpressing a BMP target gene Msx2, or in embryos in which expression of Msx2 had been activated by BMP2 and BMP4 [79, 80]. Thus, it is possible that the balance of Ski and BMP4 activity may be critical for normal craniofacial and muscle development and that the craniofacial abnormalities found in ski-null mice are due to lack of repression of BMP signaling.

Apart from the antagonistic activity of SnoN on TGF- $\beta$ signaling, one recent study suggested surprisingly that at very low concentrations SnoN may also serve as a cell type-specific co-activator of TGF- $\beta$ signaling in a mink lung epithelial cell line [75]; and this activity may be mediated by the interaction with ING2 [81]. Whether this is true for other cell lines and the biological relevance of these findings remain to be determined.

\section{Stabilization of 553 by SnoN through binding to PML}

Our recent study has revealed a novel functional pathway of $\mathrm{SnoN}$ in regulation of p53 expression (Figure 2). SnoN can interact with the PML protein through residues 322-366 immediately following the Smad4 binding domain and is recruited to the PML nuclear bodies. This results in stabilization of $\mathrm{p} 53$ and premature senescence [60]. The activity of SnoN to induce senescence is independent of its ability to bind to the Smad proteins and an- tagonize TGF- $\beta$ signaling but is associated with elevated SnoN expression. Mutant SnoN that is defective in binding to the Smad proteins associates with PML, stabilizes p53 and induces senescence as efficiently as WT SnoN. These data reveal a Smad-independent function of SnoN and provide a mechanism for SnoN function as a tumor suppressor.

\section{Other partners of Ski and SnoN}

Other cellular partners of Ski generally fall into two major categories. The first group includes members of the transcriptional repressor complex. Ski and SnoN can interact with $\mathrm{N}-\mathrm{CoR}$ and silencing mediator of retinoid and thyroid hormone receptors (SMRT), members of HDAC complexes [12, 68, 82], and bridge the repressor complex to Smads $[63,64]$, thyroid hormone receptor and $\mathrm{Mad}$ [12], or Gli3 [67] to mediate transcriptional repression by these proteins. Transcriptional repression by Mad may also involve a physical interaction between Ski and PML [83]. In transfected cells, Ski but not SnoN has also been reported to interact with $\mathrm{mSin} 3 \mathrm{~A}$, a general co-repressor involved in HDAC complex recruitment [12], HIPK2 (homeodomain-interacting protein kinase 2), another co-repressor necessary for Ski-dependent negative regulation of BMP-induced transcription [84], and MeCP2 (Methyl CpG binding protein 2) [85]. However, these interactions have not been shown with endogenous proteins, and whether they play a role in transcriptional repression by Ski is not clear.

The second group of Ski-binding partners tends to be molecules involved in cell differentiation, proliferation or hormonal responses. Ski has been shown to interact with RAR $\alpha$ to block its transactivation activity $[32,47]$, with PU.1 to negatively regulate macrophage differentiation [86], with GATA1 to block its DNA-binding activity [66], with vitamin D receptor to repress vitamin D signaling [82] and with $\mathrm{pRb}$ to abrogate $\mathrm{Rb}$-mediated transcriptional repression [65]. Recently, Ski has been found to be required for TGF- $\beta$-induced proliferation of Schwann cells, possibly by inactivating $\mathrm{Rb}$ through promoting hyperphosphorylation and cytoplasmic translocation of $\mathrm{Rb}$ [87].

Ski has been shown to activate myogenic differentiation by modulating transcriptional activity of the MyoD complex at the myogenin promoter and by inhibiting HDAC activity [28]. This mechanism may be related to the ability of Ski to promote muscle development in vivo. Finally, Ski can activate $\mathrm{Wnt} / \beta$-catenin signaling in melanoma cells by interacting with FHL2 [44]. Another transcriptional activator, SKIP (Ski interacting protein), has been reported to bind to Ski and antagonize its transcriptional co-repressor activity [88], thereby releasing molecules targeted by Ski, such as Smads or pRb [89, 
90]. It is worth noting that all the interactions mentioned above are described in transfected cells under overexpression conditions. They have yet to be confirmed in vivo and their biological significance under physiological conditions has yet to be determined.

Some of the Ski partners described above have not been shown to bind to SnoN. Similarly, not all the events regulated by SnoN are shared by Ski. For example, SnoN but not Ski has been reported to co-operate with p53 to repress transcription from the alpha-fetoprotein (AFP) promoter [91]. SnoN but not Ski has been shown to inhibit the RhoA small GTPase activity [55]. Thus, Ski and SnoN do not necessarily perform redundant functions in cells.

\section{Regulation of Ski and SnoN expression}

As both overexpression and downregulation of Ski or SnoN have been linked to cancer progression, the expression levels of both proteins need to be tightly controlled. Regulation of Ski and SnoN expression occurs at multiple levels, including transcriptional regulation, protein degradation, post-translational modifications and subcellular localization.

\section{Transcriptional regulation}

The best studied inducer of SnoN transcription is TGF- $\beta$. SnoN transcription is upregulated after $2 \mathrm{~h}$ of TGF- $\beta$ treatment through a direct binding of the Smad2/ Smad4 complex to the Smad-binding elements (SBE) in the sno promoter [63]. This upregulation of SnoN expression may function as a negative feedback mechanism to turn off TGF- $\beta$ signaling or may perform other functions including promoting transformation of fibroblasts by TGF- $\beta$ [92]. Hepatocyte Growth Factor/Scatter Factor (HGF/SF) can upregulate SnoN expression in proximal tubular kidney epithelial cells (HKC-8) [93, 94], but not in glomerular mesangial cells and interstitial fibroblasts [94, 95], indicating that $\mathrm{HGF} / \mathrm{SF}$ may be a cell typespecific activator of SnoN expression. This activation appears to involve a concerted binding of CREB and Sp1 to their cognate cis-acting elements in the sno promoter in response to HGF/SF stimulation [94]. Upregulation of SnoN expression has also been observed during liver regeneration [35], possibly mediated by HGF/SF.

In contrast to SnoN, Ski has not been shown to be a direct transcriptional target of TGF- $\beta$ signaling. In one recent report, low-dose TGF- $\beta(25 \mathrm{pg} / \mathrm{mL})$ was found to induce a slight increase in Ski level in skin fibroblasts, but high-dose TGF- $\beta(25 \mathrm{ng} / \mathrm{mL})$ repressed it [96]. It is not clear whether this regulation occurs at the level of transcription or protein stability and whether this is a di- rect effect of TGF- $\beta$ signaling.

\section{Protein degradation}

Both SnoN and Ski are regulated at the level of protein stability. Earlier experiments showed that SnoN has a half-life of approximately $4 \mathrm{~h}$ in the absence of stimuli, and within $30 \mathrm{~min}$ of TGF- $\beta$ stimulation this is shortened to around $45 \mathrm{~min}[63,97]$. Shortly after, SnoN was found to be targeted by the ubiquitin-dependent proteasome. Several E3 ubiquitin ligases have been shown to be recruited to SnoN for its ubiquitination in response to TGF- $\beta$, including Smurf2 (Smad ubiquitin regulatory factor), a HECT domain-containing E3 ubiquitin ligase [98], the Anaphase Promoting Complex (APC/ C), a RING finger ubiquitin ligase complex [99-101], and Arkadia, a RING domain-containing E3 ligase [102, 103]. These molecules interact with the R-Smads, Smad 2 and Smad3, and are recruited to SnoN to promote its ubiquitination and degradation. Stable interaction of SnoN with the APC/C complex also requires the Destruction box (D box), present in the DHD of SnoN, that is recognized and bound by $\mathrm{CDH} 1$, the targeting subunit of $\mathrm{APC} / \mathrm{C}$. Three lysine residues in the $\mathrm{C}$-terminal domain of SnoN, K440, K446 and K449, have been shown to be necessary for SnoN ubiquitination [100]. As these 3 residues are located in the exon that is not present in SnoN2, SnoN2 could be a natural variant of SnoN that is resistant to ubiquitin-dependent degradation.

Given that SnoN is expressed in virtually all cell types, the rapid degradation of SnoN in response to TGF- $\beta$ stimulation releases its repression of the Smad heteromeric complexes and allows activation of TGF- $\beta$ target genes. In human esophageal cancer cells, a truncated SnoN, which cannot be degraded accumulates to a high level in cells, resulting in resistance to TGF- $\beta$ induced growth arrest [104]. This finding highlights the importance of SnoN stability in regulating cell transformation. Ubiquitin-dependent degradation of SnoN may also play important roles in other physiological and pathological processes. For instance, Smad2-dependent degradation of SnoN through the CDH1/APC/C pathway inhibits axonal growth [30, 105]. Regulation of SnoN expression in obstructive nephropathy has been shown to also involve the ubiquitin-proteasome pathway [36, 37].

Unlike SnoN, TGF- $\beta$-induced degradation of Ski is not observed in all cell types. An earlier study showed that Ski is degraded in response to TGF- $\beta$ in mink lung epithelial cells [97]. However this was not observed in primary ovarian epithelial cells or ovarian cancer cell lines [106]. We recently showed that in metastatic breast cancer and melanoma cell lines, Ski is effectively degraded by TGF- $\beta$ signaling via Arkadia, which interacts 
with Ski through Smad2 and Smad3 to mediate its ubiquitination and degradation $[62,102]$. Since As TGF- $\beta$ is a potent promoter of breast cancer and melanoma metastasis, and Ski can inhibit breast cancer metastasis [55], this degradation of Ski induced by TGF- $\beta$ may play an important role in malignant progression of these cancer cells. So far, the lysine residues necessary for ubiquitination have not been mapped in Ski, and those lysines targeted by ubiquitination in SnoN are not conserved in Ski. Despite the fact that Ski also contains the D Box motif, the CDH1-APC complex has not been shown to bind to and downregulate Ski.

It has been observed that the expression of SnoN and Ski is regulated throughout the cell cycle. Although some data suggest that SnoN expression is higher in G1 [3, 29], others argue that the relative level of Ski and SnoN is higher in $\mathrm{G} 2 / \mathrm{M}[99,107,108]$. The cellular machinery responsible for the cell cycle-dependent regulation of these two proteins has not been identified although it has been suggested that the APC/C complex may be responsible for modulating SnoN expression [99], while cdc34 may regulate Ski expression [107] during the cell cycle independent of TGF- $\beta$ signaling.

\section{Other post-translational modifications}

SnoN can be modified by SUMOylation on lysines 50 and 383 through the action of the SUMO E3 ligase PIASs in a manner independent of TGF- $\beta$ signaling [109, 110]. SUMOylation of SnoN is independent of its ubiquitination and does not alter its stability or subcellular localization, but may potentiate SnoN-mediated repression of TGF- $\beta$ signaling on specific promoters [110]. Importantly, mutant SnoN, which cannot be SUMOylated potently activates muscle-specific gene expression and enhances myotube formation. Therefore, SUMOylation of SnoN may block its myogenic activity $[109,110]$ by an unknown mechanism. Ski has not been reported to be modified by SUMOylation, and the two key lysine residues important for SnoN SUMOylation are not conserved in Ski.

Both Ski and SnoN have been reported to be phosphorylated. SnoN can be phosphorylated on several residues including S115, S117 and/or T119 by TAK1 (TGF- $\beta$ activated kinase) [111]. This phosphorylation may facilitate ubiquitination and degradation of SnoN. Interestingly, these residues are not conserved in Ski. Ski is found to be phosphorylated on serine residues in transfected cells [112] and can serve as a substrate of the CDC2 kinase in an in vitro kinase assay [108]. However, neither the sites of phosphorylation in Ski nor the kinases responsible for the phosphorylation have been determined. The physiological significance of Ski phosphorylation is not clear.

\section{Subcellular localization}

Both Ski and SnoN were identified as nuclear proteins. However, more recent work suggests that they can localize to the cytoplasm under various conditions. In normal tissues and non-tumorigenic or primary epithelial cells, SnoN is predominantly expressed in the cytoplasm, while in malignant tissues and cell lines, it is exclusively nuclear [113]. The cytoplasmic SnoN is resistant to TGF$\beta$-induced degradation and inhibits TGF- $\beta$ signaling through sequestration of the Smads in the cytoplasm. In mammary epithelial cells, SnoN can translocate to the nucleus upon morphological differentiation or cell cycle arrest. Two lysine residues, Lys30 and Lys31, have been shown to be necessary for SnoN nuclear localization. These residues may be subjected to post-translational modifications or be involved in interaction with yet-tobe-identified partners that regulate SnoN nuclear import/ export. Ski has been reported to be predominantly localized in the nucleus [38], while several reports described cytoplasmic localization of Ski in malignant tumor cells $[40,114]$. A nuclear localization sequence has been mapped in Ski (residues 452-458) that is not conserved in SnoN [115]. Cytoplasmic Ski inhibits Smad activity but does not activate the myogenin promoter, suggesting that subcellular localization of Ski differentially regulates its activity [115]. A cytoplasmic protein, C184M, has been shown to sequester Ski in the cytoplasm when overexpressed [116]. However, whether this protein is required for the cytoplasmic localization of Ski in metastatic cancer cells in vivo is not clear. Given that their nuclear localization sequences are not shared and conserved, it is likely that subcellular localization of Ski and $\mathrm{SnoN}$ is regulated through different mechanisms.

\section{Conclusion and future directions}

Studies in the past few years have uncovered mechanisms that regulate Ski and SnoN expression at the level of transcription, protein stability or intracellular localization, and have defined the interaction between SnoN/ Ski and the Smads through structural and functional studies. Many interacting partners of Ski and SnoN have been identified. As we learn more about their functions, the complex roles of Ski and SnoN in regulation of mammalian epithelial cell function, transformation and vertebrate development are beginning to be revealed. In mammalian tumorigenesis, both pro-oncogenic and anti-oncogenic activities have been appreciated for these two proteins. Some but not all of these activities are dependent on the interaction between Ski/SnoN and the Smad proteins. A goal in the near future is to identify and characterize Smad-independent intracellular pathways 
that may be targeted by Ski/SnoN. While much attention in the past has been devoted to revealing the roles of Ski/ SnoN in human cancer cells, little is known about their functions in normal mammalian epithelial cells. This is a major challenge that deserves more research efforts in the future.

\section{References}

1 Li Y, Turck CM, Teumer JK, Stavnezer E. Unique sequence, ski, in Sloan-Kettering avian retroviruses with properties of a new cell-derived oncogene. J Virol 1986; 57:1065-1072.

2 Nomura N, Sasamoto S, Ishii S, Date T, Matsui M, Ishizaki $\mathrm{R}$. Isolation of human cDNA clones of ski and the ski-related gene, sno. Nucleic Acids Res 1989; 17:5489-5500.

3 Pearson-White S, Crittenden R. Proto-oncogene sno expression, alternative isoforms and immediate early serum response. Nucleic Acids Res 1997; 25:2930-2937.

4 Pelzer T, Lyons GE, Kim S, Moreadith RW. Cloning and characterization of the murine homolog of the sno protooncogene reveals a novel splice variant. Dev Dyn 1996; 205:114-125.

5 Pearson-White S. SnoI, a novel alternatively spliced isoform of the ski protooncogene homolog, sno. Nucleic Acids Res 1993; 21:4632-4638.

6 Arndt S, Poser I, Schubert T, Moser M, Bosserhoff AK. Cloning and functional characterization of a new Ski homolog, Fussel-18, specifically expressed in neuronal tissues. Lab Invest; J Tech Methods Pathol 2005; 85:1330-1341.

7 Arndt S, Poser I, Moser M, Bosserhoff AK. Fussel-15, a novel Ski/Sno homolog protein, antagonizes BMP signaling. Mol Cell Neurosci 2007; 34:603-611.

8 Kozmik Z, Pfeffer P, Kralova J, et al. Molecular cloning and expression of the human and mouse homologues of the Drosophila dachshund gene. Dev Genes Evol 1999; 209:537545.

9 Wu K, Yang Y, Wang C, et al. DACH1 inhibits transforming growth factor-beta signaling through binding Smad4. J Biol Chem 2003; 278:51673-51684.

10 Wu JW, Krawitz AR, Chai J, et al. Structural mechanism of Smad4 recognition by the nuclear oncoprotein Ski: insights on Ski-mediated repression of TGF-beta signaling. Cell 2002; 111:357-367.

11 Wilson JJ, Malakhova M, Zhang R, Joachimiak A, Hegde RS. Crystal structure of the dachshund homology domain of human SKI. Structure 2004; 12:785-792.

12 Nomura T, Khan MM, Kaul SC, et al. Ski is a component of the histone deacetylase complex required for transcriptional repression by Mad and thyroid hormone receptor. Genes Dev 1999; 13:412-423.

13 Heyman HC, Stavnezer E. A carboxyl-terminal region of the ski oncoprotein mediates homodimerization as well as heterodimerization with the related protein SnoN. J Biol Chem 1994; 269:26996-27003.

14 Cohen SB, Zheng G, Heyman HC, Stavnezer E. Heterodimers of the SnoN and Ski oncoproteins form preferentially over homodimers and are more potent transforming agents. Nucleic Acids Res 1999; 27:1006-1014.
15 Kaufman CD, Martinez-Rodriguez G, Hackett PB Jr. Ectopic expression of c-ski disrupts gastrulation and neural patterning in zebrafish. Mech Dev 2000; 95:147-162.

16 Berk M, Desai SY, Heyman HC, Colmenares C. Mice lacking the ski proto-oncogene have defects in neurulation, craniofacial, patterning, and skeletal muscle development. Genes Dev 1997; 11:2029-2039.

17 Amaravadi LS, Neff AW, Sleeman JP, Smith RC. Autonomous neural axis formation by ectopic expression of the protooncogene c-ski. Dev Biol 1997; 192:392-404.

18 Sleeman JP, Laskey RA. Xenopus c-ski contains a novel coiled-coil protein domain, and is maternally expressed during development. Oncogene 1993; 8:67-77.

19 Lyons GE, Micales BK, Herr MJ, et al. Protooncogene c-ski is expressed in both proliferating and postmitotic neuronal populations. Dev Dyn 1994; 201:354-365.

20 Namciu S, Lyons GE, Micales BK, Heyman HC, Colmenares C, Stavnezer E. Enhanced expression of mouse c-ski accompanies terminal skeletal muscle differentiation in vivo and in vitro. Dev Dyn 1995; 204:291-300.

21 Gajecka M, Mackay KL, Shaffer LG. Monosomy 1p36 deletion syndrome. Am J Med Genet 2007; 145C:346-356.

22 Colmenares C, Heilstedt HA, Shaffer LG, et al. Loss of the SKI proto-oncogene in individuals affected with 1 p36 deletion syndrome is predicted by strain-dependent defects in Ski-/- mice. Nat Genet 2002; 30:106-109.

23 Shinagawa T, Dong HD, Xu M, Maekawa T, Ishii S. The sno gene, which encodes a component of the histone deacetylase complex, acts as a tumor suppressor in mice. EMBO J 2000; 19:2280-2291.

24 Pearson-White S, McDuffie M. Defective T-cell activation is associated with augmented transforming growth factor Beta sensitivity in mice with mutations in the Sno gene. Mol Cell Biol 2003; 23:5446-5459.

25 Boyer PL, Colmenares C, Stavnezer E, Hughes SH. Sequence and biological activity of chicken snoN cDNA clones. Oncogene 1993; 8:457-466.

26 Colmenares C, Stavnezer E. The ski oncogene induces muscle differentiation in quail embryo cells. Cell 1989; 59:293303.

27 Sutrave P, Kelly AM, Hughes SH. Ski can cause selective growth of skeletal muscle in transgenic mice. Genes Dev 1990; 4:1462-1472.

28 Kobayashi N, Goto K, Horiguchi K, et al. c-Ski activates MyoD in the nucleus of myoblastic cells through suppression of histone deacetylases. Genes Cells 2007; 12:375-385.

29 Mimura N, Ichikawa K, Asano A, Nagase T, Ishii S. A transient increase of snoN transcript by growth arrest upon serum deprivation and cell-to-cell contact. FEBS Lett 1996; 397:253-259.

30 Stegmuller J, Konishi Y, Huynh MA, Yuan Z, Dibacco S, Bonni A. Cell-intrinsic regulation of axonal morphogenesis by the Cdh1-APC target SnoN. Neuron 2006; 50:389-400.

31 Atanasoski S, Notterpek L, Lee HY, et al. The protooncogene Ski controls Schwann cell proliferation and myelination. Neuron 2004; 43:499-511.

32 Dahl R, Kieslinger M, Beug H, Hayman MJ. Transformation of hematopoietic cells by the Ski oncoprotein involves 
repression of retinoic acid receptor signaling. Proc Natl Acad Sci USA 1998; 95:11187-11192.

33 Pearson-White S, Deacon D, Crittenden R, et al. The ski/sno protooncogene family in hematopoietic development. Blood 1995; 86:2146-2155.

34 Liu X, Zhang E, Li P, et al. Expression and possible mechanism of c-ski, a novel tissue repair-related gene during normal and radiation-impaired wound healing. Wound Repair Regen 2006; 14:162-171.

35 Macias-Silva M, Li W, Leu JI, Crissey MA, Taub R. Upregulated transcriptional repressors SnoN and Ski bind Smad proteins to antagonize transforming growth factor-beta signals during liver regeneration. $J$ Biol Chem 2002; 277:2848328490.

36 Tan R, Zhang J, Tan X, Zhang X, Yang J, Liu Y. Downregulation of SnoN expression in obstructive nephropathy is mediated by an enhanced ubiquitin-dependent degradation. $J \mathrm{Am}$ Soc Nephrol 2006; 17:2781-2791.

37 Fukasawa H, Yamamoto T, Togawa A, et al. Ubiquitin-dependent degradation of SnoN and Ski is increased in renal fibrosis induced by obstructive injury. Kidney Int 2006; 69:17331740 .

38 Colmenares C, Sutrave P, Hughes SH, Stavnezer E. Activation of the c-ski oncogene by overexpression. J Virol 1991; 65:4929-4935.

39 Imoto I, Pimkhaokham A, Fukuda Y, et al. SNO is a probable target for gene amplification at 3q26 in squamous-cell carcinomas of the esophagus. Biochem Biophys Res Commun 2001; 286:559-565.

40 Fukuchi M, Nakajima M, Fukai Y, et al. Increased expression of c-Ski as a co-repressor in transforming growth factor-beta signaling correlates with progression of esophageal squamous cell carcinoma. Int J Cancer 2004; 108:818-824.

41 Akagi I, Miyashita M, Makino H, et al. SnoN overexpression is predictive of poor survival in patients with esophageal squamous cell carcinoma. Ann Surg Oncol 2008; 15:29652975

42 Poser I, Rothhammer T, Dooley S, Weiskirchen R, Bosserhoff AK. Characterization of Sno expression in malignant melanoma. Int J Oncol 2005; 26:1411-1417.

43 Reed JA, Lin Q, Chen D, Mian IS, Medrano EE. SKI pathways inducing progression of human melanoma. Cancer Metastasis Rev 2005; 24:265-272.

44 Chen D, Xu W, Bales E, et al. SKI activates Wnt/beta-catenin signaling in human melanoma. Cancer Res 2003; 63:66266634.

45 Zhang F, Lundin M, Ristimaki A, et al. Ski-related novel protein $\mathrm{N}$ (SnoN), a negative controller of transforming growth factor-beta signaling, is a prognostic marker in estrogen receptor-positive breast carcinomas. Cancer Res 2003; 63:5005-5010.

46 Buess M, Terracciano L, Reuter J, et al. Amplification of SKI is a prognostic marker in early colorectal cancer. Neoplasia (New York, NY) 2004; 6:207-212.

47 Ritter M, Kattmann D, Teichler S, et al. Inhibition of retinoic acid receptor signaling by Ski in acute myeloid leukemia. Leukemia 2006; 20:437-443.

48 Racz A, Brass N, Heckel D, Pahl S, Remberger K, Meese E. Expression analysis of genes at 3q26-q27 involved in fre- quent amplification in squamous cell lung carcinoma. Eur $J$ Cancer 1999; 35:641-646.

49 Sugita M, Tanaka N, Davidson S, et al. Molecular definition of a small amplification domain within 3q26 in tumors of cervix, ovary, and lung. Cancer Genet Cytogenet 2000; 117:918.

50 Singh B, Gogineni SK, Sacks PG, et al. Molecular cytogenetic characterization of head and neck squamous cell carcinoma and refinement of $3 \mathrm{q}$ amplification. Cancer Res 2001; 61:4506-4513.

51 Lin SC, Liu CJ, Ko SY, Chang HC, Liu TY, Chang KW. Copy number amplification of 3q26-27 oncogenes in microdissected oral squamous cell carcinoma and oral brushed samples from areca chewers. J Pathol 2005; 206:417-422.

52 Hopman AH, Theelen W, Hommelberg PP, et al. Genomic integration of oncogenic HPV and gain of the human telomerase gene TERC at 3q26 are strongly associated events in the progression of uterine cervical dysplasia to invasive cancer. J Pathol 2006; 210:412-419.

53 Nanjundan M, Nakayama Y, Cheng KW, et al. Amplification of MDS1/EVI1 and EVI1, located in the 3q26.2 amplicon, is associated with favorable patient prognosis in ovarian cancer. Cancer Res 2007; 67:3074-3084.

54 Jung V, Kindich R, Kamradt J, et al. Genomic and expression analysis of the 3q25-q26 amplification unit reveals TLOC1/ SEC62 as a probable target gene in prostate cancer. Mol Cancer Res 2006; 4:169-176.

55 Zhu Q, Krakowski AR, Dunham EE, et al. Dual role of SnoN in mammalian tumorigenesis. Mol Cell Biol 2007; 27:324339.

56 Heider TR, Lyman S, Schoonhoven R, Behrns KE. Ski promotes tumor growth through abrogation of transforming growth factor-beta signaling in pancreatic cancer. Ann Surg 2007; 246:61-68.

57 Villanacci V, Bellone G, Battaglia E, et al. Ski/SnoN expression in the sequence metaplasia-dysplasia-adenocarcinoma of Barrett's esophagus. Hum Pathol 2008; 39:403-409.

58 Chia JA, Simms LA, Cozzi SJ, et al. SnoN expression is differently regulated in microsatellite unstable compared with microsatellite stable colorectal cancers. BMC Cancer 2006; 6:252.

59 Shinagawa T, Nomura T, Colmenares C, Ohira M, Nakagawara A, Ishii S. Increased susceptibility to tumorigenesis of ski-deficient heterozygous mice. Oncogene 2001; 20:81008108.

60 Pan D, Zhu Q, Luo K. SnoN functions as a tumor suppressor by inducing premature senescence. Submitted.

61 Ramel MC, Emery CM, Foulger R, Goberdhan DC, van den Heuvel M, Wilson C. Drosophila SnoN modulates growth and patterning by antagonizing TGF-beta signalling. Mech Dev 2007; 124:304-317.

62 Le Scolan E, Zhu Q, Wang L, et al. Transforming growth factor-beta suppresses the ability of Ski to inhibit tumor metastasis by inducing its degradation. Cancer Res 2008; 68:32773285.

63 Stroschein SL, Wang W, Zhou S, Zhou Q, Luo K. Negative feedback regulation of TGF-beta signaling by the SnoN oncoprotein. Science (New York, NY) 1999; 286:771-774.

64 Luo K, Stroschein SL, Wang W, et al. The Ski oncoprotein 
interacts with the Smad proteins to repress TGFbeta signaling. Genes Dev 1999; 13:2196-2206.

65 Tokitou F, Nomura T, Khan MM, et al. Viral ski inhibits retinoblastoma protein $(\mathrm{Rb})$-mediated transcriptional repression in a dominant negative fashion. J Biol Chem 1999; 274:4485-4488.

66 Ueki N, Zhang L, Hayman MJ. Ski negatively regulates erythroid differentiation through its interaction with GATA1. Mol Cell Biol 2004; 24:10118-10125.

67 Dai P, Shinagawa T, Nomura T, et al. Ski is involved in transcriptional regulation by the repressor and full-length forms of Gli3. Genes Dev 2002; 16:2843-2848.

68 Karagianni P, Wong J. HDAC3: taking the SMRT-N-CoRrect road to repression. Oncogene 2007; 26:5439-5449.

69 Luo K. Ski and SnoN: negative regulators of TGF-beta signaling. Curr Opin Genet Dev 2004; 14:65-70.

70 Suzuki H, Yagi K, Kondo M, Kato M, Miyazono K, Miyazawa K. c-Ski inhibits the TGF-beta signaling pathway through stabilization of inactive Smad complexes on Smad-binding elements. Oncogene 2004; 23:5068-5076.

71 Ueki N, Hayman MJ. Direct interaction of Ski with either Smad3 or Smad4 is necessary and sufficient for Ski-mediated repression of transforming growth factor-beta signaling. $J$ Biol Chem 2003; 278:32489-32492.

72 Chen W, Lam SS, Srinath H, Schiffer CA, Royer WE Jr., Lin $\mathrm{K}$. Competition between Ski and CREB-binding protein for binding to Smad proteins in transforming growth factor-beta signaling. J Biol Chem 2007; 282:11365-11376.

73 Akiyoshi S, Inoue H, Hanai J, et al. c-Ski acts as a transcriptional co-repressor in transforming growth factor-beta signaling through interaction with smads. J Biol Chem 1999; 274:35269-35277.

74 Prunier C, Pessah M, Ferrand N, Seo SR, Howe P, Atfi A. The oncoprotein Ski acts as an antagonist of transforming growth factor-beta signaling by suppressing Smad2 phosphorylation. J Biol Chem 2003; 278:26249-26257.

75 Sarker KP, Wilson SM, Bonni S. SnoN is a cell type-specific mediator of transforming growth factor-beta responses. J Biol Chem 2005; 280:13037-13046.

76 He J, Tegen SB, Krawitz AR, Martin GS, Luo K. The transforming activity of Ski and SnoN is dependent on their ability to repress the activity of Smad proteins. J Biol Chem 2003; 278:30540-30547.

77 Takeda M, Mizuide M, Oka M, et al. Interaction with Smad4 is indispensable for suppression of BMP signaling by c-Ski. Mol Biol Cell 2004; 15:963-972.

78 Wang W, Mariani FV, Harland RM, Luo K. Ski represses bone morphogenic protein signaling in Xenopus and mammalian cells. Proc Natl Acad Sci USA 2000; 97:14394-14399.

79 Winograd J, Reilly MP, Roe R, et al. Perinatal lethality and multiple craniofacial malformations in MSX2 transgenic mice. Hum Mol Genet 1997; 6:369-379.

80 Barlow AJ, Francis-West PH. Ectopic application of recombinant BMP-2 and BMP-4 can change patterning of developing chick facial primordia. Development (Cambridge, England) 1997; 124:391-398.

81 Sarker KP, Kataoka H, Chan A, et al. ING2 as a novel mediator of transforming growth factor-beta-dependent responses in epithelial cells. J Biol Chem 2008; 283:13269-13279.
82 Ueki N, Hayman MJ. Signal-dependent N-CoR requirement for repression by the Ski oncoprotein. J Biol Chem 2003; 278:24858-24864.

83 Khan MM, Nomura T, Kim H, et al. Role of PML and PMLRARalpha in Mad-mediated transcriptional repression. Mol Cell 2001; 7:1233-1243.

84 Harada J, Kokura K, Kanei-Ishii C, et al. Requirement of the co-repressor homeodomain-interacting protein kinase 2 for ski-mediated inhibition of bone morphogenetic proteininduced transcriptional activation. J Biol Chem 2003; 278:38998-39005.

85 Kokura K, Kaul SC, Wadhwa R, et al. The Ski protein family is required for MeCP2-mediated transcriptional repression. $J$ Biol Chem 2001; 276:34115-34121.

86 Ueki N, Zhang L, Hayman MJ. Ski can negatively regulates macrophage differentiation through its interaction with PU.1. Oncogene 2008; 27:300-307.

87 Jacob C, Grabner H, Atanasoski S, Suter U. Expression and localization of Ski determine cell type-specific TGFbeta signaling effects on the cell cycle. J Cell Biol 2008; 182:519-530.

88 Dahl R, Wani B, Hayman MJ. The Ski oncoprotein interacts with Skip, the human homolog of Drosophila Bx42. Oncogene 1998; 16:1579-1586.

89 Leong GM, Subramaniam N, Figueroa J, et al. Ski-interacting protein interacts with Smad proteins to augment transforming growth factor-beta-dependent transcription. J Biol Chem 2001; 276:18243-18248.

90 Prathapam T, Kuhne C, Banks L. Skip interacts with the retinoblastoma tumor suppressor and inhibits its transcriptional repression activity. Nucleic Acids Res 2002; 30:52615268.

91 Wilkinson DS, Ogden SK, Stratton SA, et al. A direct intersection between p53 and transforming growth factor beta pathways targets chromatin modification and transcription repression of the alpha-fetoprotein gene. Mol Cell Biol 2005; 25:1200-1212.

92 Zhu Q, Pearson-White S, Luo K. Requirement for the SnoN oncoprotein in transforming growth factor beta-induced oncogenic transformation of fibroblast cells. Mol Cell Biol 2005; 25:10731-10744.

93 Yang J, Dai C, Liu Y. A novel mechanism by which hepatocyte growth factor blocks tubular epithelial to mesenchymal transition. J Am Soc Nephrol 2005; 16:68-78.

94 Tan R, Zhang X, Yang J, Li Y, Liu Y. Molecular basis for the cell type specific induction of SnoN expression by hepatocyte growth factor. J Am Soc Nephrol 2007; 18:2340-2349.

95 Dai C, Liu Y. Hepatocyte growth factor antagonizes the profibrotic action of TGF-beta1 in mesangial cells by stabilizing Smad transcriptional corepressor TGIF. J Am Soc Nephrol 2004; 15:1402-1412.

96 Liu X, Li P, Liu P, et al. The essential role for c-Ski in mediating TGF-betal-induced bi-directional effects on skin fibroblast proliferation through a feedback loop. Biochem J 2008; 409:289-297.

97 Sun Y, Liu X, Ng-Eaton E, Lodish HF, Weinberg RA. SnoN and Ski protooncoproteins are rapidly degraded in response to transforming growth factor beta signaling. Proc Natl Acad Sci USA 1999; 96:12442-12447.

98 Bonni S, Wang HR, Causing CG, et al. TGF-beta induces 
assembly of a Smad2-Smurf2 ubiquitin ligase complex that tarncer Res 2005; 65:4782-4788.

99 Wan Y, Liu X, Kirschner MW. The anaphase-promoting complex mediates TGF-beta signaling by targeting SnoN for destruction. Mol Cell 2001; 8:1027-1039.

100 Stroschein SL, Bonni S, Wrana JL, Luo K. Smad3 recruits the anaphase-promoting complex for ubiquitination and degradation of SnoN. Genes Dev 2001; 15:2822-2836.

$101 \mathrm{Wu}$ G, Glickstein S, Liu W, et al. The anaphase-promoting complex coordinates initiation of lens differentiation. Mol Biol Cell 2007; 18:1018-1029.

102 Nagano Y, Mavrakis KJ, Lee KL, et al. Arkadia induces degradation of SnoN and c-Ski to enhance transforming growth factor-beta signaling. J Biol Chem 2007; 282:20492-20501.

103 Levy L, Howell M, Das D, Harkin S, Episkopou V, Hill CS. Arkadia activates Smad3/Smad4-dependent transcription by triggering signal-induced SnoN degradation. Mol Cell Biol 2007; 27:6068-6083.

104 Edmiston JS, Yeudall WA, Chung TD, Lebman DA. Inability of transforming growth factor-beta to cause SnoN degradation leads to resistance to transforming growth factor-betainduced growth arrest in esophageal cancer cells. Cancer Res 2005; 65:4782-4788.

105 Stegmuller J, Huynh MA, Yuan Z, Konishi Y, Bonni A. TGFbeta-Smad2 signaling regulates the Cdh1-APC/SnoN pathway of axonal morphogenesis. J Neurosci 2008; 28:1961-1969.

106 Baldwin RL, Tran H, Karlan BY. Loss of c-myc repression coincides with ovarian cancer resistance to transforming growth factor beta growth arrest independent of transforming growth factor beta/Smad signaling. Cancer Res 2003; 63:1413-1419.

107 Macdonald M, Wan Y, Wang W, et al. Control of cell cycledependent degradation of c-Ski proto-oncoprotein by $\mathrm{Cdc} 34$. Oncogene 2004; 23:5643-5653.
108 Marcelain K, Hayman MJ. The Ski oncoprotein is upregulated and localized at the centrosomes and mitotic spindle during mitosis. Oncogene 2005; 24:4321-4329.

109 Wrighton KH, Liang M, Bryan B, et al. Transforming growth factor-beta-independent regulation of myogenesis by $\mathrm{SnoN}$ sumoylation. J Biol Chem 2007; 282:6517-6524.

110 Hsu YH, Sarker KP, Pot I, Chan A, Netherton SJ, Bonni S. Sumoylated SnoN represses transcription in a promoterspecific manner. J Biol Chem 2006; 281:33008-33018.

111 Kajino T, Omori E, Ishii S, Matsumoto K, Ninomiya-Tsuji J. TAK1 MAPK kinase kinase mediates transforming growth factor-beta signaling by targeting SnoN oncoprotein for degradation. J Biol Chem 2007; 282:9475-9481.

112 Sutrave P, Copeland TD, Showalter SD, Hughes SH. Characterization of chicken c-ski oncogene products expressed by retrovirus vectors. Mol Cell Biol 1990; 10:3137-3144.

113 Krakowski AR, Laboureau J, Mauviel A, Bissell MJ, Luo K. Cytoplasmic SnoN in normal tissues and nonmalignant cells antagonizes TGF-beta signaling by sequestration of the Smad proteins. Proc Natl Acad Sci USA 2005; 102:12437-12442.

114 Reed JA, Bales E, Xu W, Okan NA, Bandyopadhyay D, Medrano EE. Cytoplasmic localization of the oncogenic protein Ski in human cutaneous melanomas in vivo: functional implications for transforming growth factor beta signaling. Cancer Res 2001; 61:8074-8078.

115 Nagata M, Goto K, Ehata S, et al. Nuclear and cytoplasmic c-Ski differently modulate cellular functions. Genes Cells 2006; 11:1267-1280.

116 Kokura K, Kim H, Shinagawa T, Khan MM, Nomura T, Ishii $\mathrm{S}$. The Ski-binding protein $\mathrm{C} 184 \mathrm{M}$ negatively regulates tumor growth factor-beta signaling by sequestering the Smad proteins in the cytoplasm. J Biol Chem 2003; 278:2013320139. 